\title{
Kelvin-Helmholtz instability of kink waves in photospheric twisted flux tubes
}

\author{
I. Zhelyazkov ${ }^{1}$ and T. V. Zaqarashvili²,3 \\ 1 Faculty of Physics, Sofia University, 5 James Bourchier Blvd., 1164 Sofia, Bulgaria \\ e-mail: izh@phys.uni-sofia.bg \\ 2 Space Research Institute, Austrian Academy of Sciences, Schmiedlstrasse 6, 8042 Graz, Austria \\ e-mail: teimuraz.zaqarashvili@oeaw.ac.at \\ 3 Abastumani Astrophysical Observatory at Ilia State University, 2 University Street, 0160 Tbilisi, Georgia
}

Received 30 April 2012 / Accepted 11 September 2012

\begin{abstract}
Aims. We investigate conditions under which kink magnetohydrodynamic waves propagating along photospheric uniformly twisted flux tubes with axial mass flows become unstable as a consequence of the Kelvin-Helmholtz instability.

Methods. We employed the dispersion relations of kink waves derived from the linearised magnetohydrodynamic equations. We assumed real wave numbers and complex angular wave frequencies, namely complex wave phase velocities. The dispersion relations were solved numerically at fixed input parameters and several mass flow velocities.

Results. We show that the stability of the waves depends upon four parameters, the density contrast between the flux tube and its environment, the ratio of the background magnetic fields in the two media, the twist of the magnetic field lines inside the tube, and the value of the Alfvén-Mach number (the ratio of the jet velocity to Alfvén speed inside the flux tube). We assume that the azimuthal component of the magnetic field in the tube is proportional to the distance from the tube axis and that the tube is only weakly twisted (i.e., the ratio of the azimuthal and axial components of the magnetic field is low). At certain densities and magnetic field twists, an instability of the Kelvin-Helmholtz type of kink $(m=1)$ mode can arise if the Alfvén-Mach number exceeds a critical value. In particular, for an isolated twisted magnetic flux tube (magnetically free environment) at a density contrast (the ratio of the mass density of the environment to that of the tube itself) equal to 2 and a magnetic field twist (defined as the ratio of azimuthal magnetic field component at the inner surface of the tube to the background magnetic field strength) equal to 0.4, the threshold Alfvén-Mach number has a magnitude of 1.250075 , which means that for an Alfvén speed inside the tube of $10 \mathrm{~km} \mathrm{~s}^{-1}$ the jet velocity should be higher than $12.5 \mathrm{~km} \mathrm{~s}^{-1}$ to ensure the onset of the Kelvin-Helmholtz instability of the kink $(m=1)$ mode. Speeds of that order can be detected in photospheric tubes.

Conclusions. The observed mass flows may trigger the Kelvin-Helmholtz instability of the kink $(m=1)$ mode in weakly twisted photospheric magnetic flux tubes at critical Alfvén-Mach numbers lower that those in untwisted tubes if the magnetic field twist lies in the range 0.36-0.4 and the flow speed exceeds a critical value. A weak external magnetic field (with a ratio to the magnetic field inside the tube in the range $0.1-0.5$ ) slightly increases that critical value.
\end{abstract}

Key words. magnetohydrodynamics (MHD) - waves - plasmas - magnetic fields - methods: numerical

\section{Introduction}

With the wealth of recent high-resolution observations from SDO, Hinode, Stereo, TRACE, SOHO, and RHESSI as well as from ground-based observations, the concepts of the magnetic flux tube and coronal loops have become increasingly important for understanding explosive phenomena such as solar flares and eruptive prominences and also for the problem of the solar corona heating mechanism. The observations reveal a wide range of shapes and sizes, ranging from the small-size magnetic flux tubes found in the granular network, sunspots, coronal loops, and arcades with many loops to huge loops such as prominences. The registered mass flows speeds in these magnetic tubes range from a few tens of kilometers per second in the photosphere/chromosphere (Shibata et al. 2007; Katsukawa et al. 2007; De Pontieu et al. 2007) up to hundreds or thousands of kilometers per second in coronal holes and X-ray jets (Shimojo \& Shibata 2000; Cirtain et al. 2007; Ofman \& Wang 2008; Wallace et al. 2010; Madjarska 2011; Kamio et al. 2011; Tian et al. 2011; Srivastava \& Murawski 2011).
An additional feature of the flux tube is its twist. There is observational evidence for a twist in the solar atmosphere. Aschwanden et al. (1999) confirmed the existence of twisted loops in the corona by detecting a post-flare loop system in extreme ultraviolet wavelengths ( $171 \AA$ ) with the Coronal Explorer (TRACE). Rotational movement along a loop as observed in the active region NOAA 8668 of the photosphere by Chae et al. (2001) indicates that there is a twist in the magnetic field lines. More recently, Srivastava et al. (2010), using multiwavelength observations of SOHO/MDI, SOT-Hinode/bluecontinuum (4504 $\AA$ ), G band (4305 $\AA$ ), Ca II H (3968 $)$ ), and TRACE $171 \AA$, presented the observational signature of a strongly twisted magnetic loop in AR 10960 during the period 04:43-04:52 UT on June 4, 2007.

It is well-known that the hydromagnetic flows are generally unstable against the Kelvin-Helmholtz instability when the flow speed exceeds a certain threshold/critical value (Chandrasekhar 1961). Recent observations of Kelvin-Helmholtz vortices (Foullon et al. 2011; Ofman \& Thompson 2011) increased the 
interest in flow instability in the solar corona. On the other hand, the twisted magnetic field itself can cause the so-called kink instability even when there is no flow. The stability of static twisted magnetic flux tubes has also been studied extensively in the context of laboratory plasma (e.g., Shafranov 1957; Kruskal et al. 1958). Magnetic tubes are subject to the kink instability when the twist exceeds a critical value (Lundquist 1951; Hood \& Priest 1979). Oscillations and waves and their stability in twisted magnetic flux tubes without flow have been studied in the framework of the normal mode analysis in earlier works (see Dungey \& Loughhead 1954; Roberts 1956; Trehan 1958; Bogdan 1984; Linton et al. 1996; Bennett et al. 1999; Erdélyi \& Carter 2006; Erdélyi \& Fedun 2006, 2007, 2010; Ruderman 2007; Carter \& Erdélyi 2008; Ruderman \& Erdélyi 2009; Karami \& Barin 2009; and Karami \& Bahari 2010). Most of these papers deal with relatively simple twisted magnetic configurations: incompressible plasma cylinders/slabs surrounded by perfectly conducting unmagnetised plasma or a medium with an untwisted homogeneous magnetic field. Erdélyi \& Fedun (2006) were the first to study the wave propagation in a twisted cylindrical magnetic flux tube embedded in an incompressible but also magnetically twisted plasma. A more complex magnetic field configuration has been studied by Erdélyi \& Carter (2006), who investigated the propagation of magnetohydrodynamic (MHD) waves in a fully magnetically twisted flux tube consisting of a core, an annulus, and an external region. These authors considered magnetic twist just in the annulus, while the internal and external regions retained a straight magnetic field. Two modes of sausage oscillations occur in this configuration, notably pure surface (i.e., evanescent) and hybrid (spatially oscillatory in the twisted annulus, otherwise evanescent) waves. The propagation characteristics of the kink mode in the same magnetically twisted annulus have been studied by Carter \& Erdélyi (2008). The influence of compressibility on the dispersion characteristics of the sausage mode $(m=0)$ propagating in a magnetically twisted flux tube embedded in a compressible uniformly magnetised plasma environment in cylindrical geometry has been investigated by Erdélyi \& Fedun (2007). That analysis has been generalised by the authors (see Erdélyi \& Fedun 2010) for the kink mode $(m=1)$, as well as for the fluting modes $(m>1)$; $m$ being the azimuthal order of a given mode. The last work by Erdélyi \& Fedun (2010) - the most elaborate study to date deals with the MHD wave propagation along twisted magnetic tubes of compressible ideal plasma surrounded by also compressible but uniformly magnetised fully ionized medium. The effect of a twisted magnetic field on the spectra and the resonant absorption of kink and fluting surface modes in resistive incompressible cylindrical plasmas have been studied by Karami \& Barin (2009) and Karami \& Bahari (2010). It was found that a magnetic twist will increase the frequencies, damping rates, and the ratio of the oscillation frequency to the damping rate of these modes. The period ratio $P_{1} / P_{2}$ of the fundamental and its first-overtone surface waves for kink $(m=1)$ and fluting $(m=2,3)$ modes is lower than two (the value for an untwisted loop) in the presence of a twisted magnetic field. In particular, for the kink modes, the magnetic twists $B_{\phi} / B_{z}=0.0065$ and 0.0255 can achieve deviations from two of the same order of magnitude as in the observations. The only work studying the wave propagation in a twisted magnetic tube with a mass density variation along the tube is that of Ruderman (2007). He investigated non-axisymmetric oscillations of thin (tube radius $a$ much smaller than the finite tube length $L$ ) twisted magnetic tubes in a zero-beta plasma. With an asymptotic analysis, Ruderman (2007) showed that the eigenmodes and the eigenfrequencies of the kink and fluting oscillations are described by a classical Sturm-Liouville problem for a second-order ordinary differential equation. He also concluded that the results concerning non-axisymmetric waves in twisted magnetic tubes obtained by Bennett et al. (1999) for incompressible plasmas can be applied to global non-axisymmetric modes, i.e., kink and fluting modes, in coronal loop even though the coronal plasma is a low-beta plasma. This conclusion also agrees well with the results obtained by Erdélyi \& Fedun (2007).

An open question is how a flow along a twisted magnetic flux tube will change the dispersion properties of the propagating modes and their stability. It turns out that the flow may decrease the threshold for the kink instability, as was tested experimentally in a laboratory twisted plasma column (Furno et al. 2007). This observation was theoretically confirmed by Zaqarashvili et al. (2010). The authors studied the influence of axial mass flows on the stability of an isolated twisted magnetic tube of incompressible plasma embedded in a perfectly conducting unmagnetised plasma. Two main results were found. First, the axial mass flow reduces the threshold of the kink instability in twisted magnetic tubes. Second, the twist of the magnetic field leads to the Kelvin-Helmholtz instability of sub-Alfvénic flows for the harmonics with a sufficiently large azimuthal mode number $m$. The effect is more significant for photospheric magnetic flux tubes than for coronal ones. In a recent paper, Díaz et al. (2011) also studied the equilibrium and stability of twisted magnetic flux tubes with mass flows, but for flows along the field lines. The authors focused on the stability and oscillatory modes of magnetic tubes with a uniform twist in a zero-beta plasma surrounded by a uniform cold plasma embedded in a purely longitudinal magnetic filed. Regarding the equilibrium, the authors claimed that the only value of the flow that satisfies the equations for their magnetic field configuration is a super-Alfvénic one. The main conclusion is that the twisted tube is subject to the kink instability unless the magnetic field pitch is very high, since the Lundquist criterion is significantly lowered. This is caused by the requirement of having an Alfvén-Mach number greater than 1 , so the magnetic pressure balances the magnetic field tension and fluid inertia. The authors suggest that this type of instability might be observed in some solar atmospheric structures, such as surges.

Kink waves are frequently observed in the chromospheric spectral lines as a periodic Doppler shift and/or periodic spatial displacement of the tube axis (Kukhianidze et al. 2006; Zaqarashvili et al. 2007; Zaqarashvili \& Erdélyi 2009; He et al. 2009; Pietarila et al. 2011; Tavabi et al. 2011; Ebadi et al. 2012). Most of the observations have been associated to spicules, which are chromospheric plasma jets. It is well known that a simple hydrodynamic jet is unstable against the antisymmetric (kink) mode (Drazin 2002; Zaqarashvili 2011), therefore the observed periodic displacements can be connected to the unstable kink mode of a plasma jet in spicules. However, the magnetic field of spicules may significantly influence the stability of the kink mode in the plasma jet, therefore studying the instability of $m=1$ mode of the jet in a magnetic cylinder is of vital importance.

In the present work, our aim is to investigate the effect of a twisted magnetic field on the stability of kink ( $m=1)$ waves propagating on a cylindrical incompressible radially homogeneous tube with axial mass flow, assuming that the waves are subject, under certain conditions, to the Kelvin-Helmholtz instability. To evaluate that effect, we compare the critical flow speeds at which the instability occurs with those in the untwisted magnetic tubes. It is necessary to point out that our simplified 
model of radially homogeneous plasma density inside the tube rules out the resonant absorption of MHD modes that can modify both the wave frequency spectrum and conditions under which the Kelvin-Helmholtz instability occurs. The radial density inhomogeneity is usually introduced as a nonuniform transition layer that continuously connects the internal density to the external one. Owing to this transverse inhomogeneous transitional layer, wave modes with $m \neq 1$ are spatially damped by resonant absorption. In particular, the effect of longitudinal flow on the resonantly damped propagating kink waves has been studied by Terradas et al. (2010a, 2010b) and by Soler et al. (2010a). We note that a Kelvin-Helmholtz instability of kink oscillations in flux tubes with both a sharp and smooth transition layer can also occur as a result of shear motions (Terradas et al. 2008). But as Terradas et al. (2008) claim, a magnetic twist, not included in their model, might decrease or even suppress the instability because a magnetic field component along the flow stabilises the Kelvin-Helmholtz instability. The same conclusion for the stabilising role of a small azimuthal component of the magnetic field has been drawn in the paper by Soler et al. (2010b). The influence of a twisted magnetic field on the stability status of kink waves propagating along radially inhomogeneous solar cylindrical photospheric/chromospheric jets is beyond the scope of this study, and as was mentioned above, we limit ourselves to considering radially homogeneous flowing plasmas only.

This paper is organized as follows. In Sect. 2 we specify the geometry of the problem, governing equations, and the derivation of the wave dispersion relation. Section 3 deals with the numerical solutions to the dispersion relation for various values of the input parameters and compares derived dispersion curves and growth rates of the unstable kink waves with those in untwisted magnetic tubes. The last Sect. 4 summarises our results.

\section{Geometry, the basic MHD equations, and the wave dispersion relation}

We consider a magnetic flux tube with radius $a$ and density $\rho_{\mathrm{i}}$ embedded in a uniform field environment with density $\rho_{\mathrm{e}}$. Both media (inside and outside the tube) are supposed to be incompressible. The magnetic field inside the tube is helicoidal, $\boldsymbol{B}_{\mathrm{i}}=\left(0, B_{\mathrm{i} \phi}(r), B_{\mathrm{i} z}(r)\right)$, while outside the magnetic field is uniform and directed along the $z$-axis, $\boldsymbol{B}_{\mathrm{e}}=\left(0,0, B_{\mathrm{e}}\right)$. Both $\rho_{\mathrm{i}}$ and $\rho_{\mathrm{e}}$ are assumed to be homogeneous. We consider the mass flow $\boldsymbol{v}_{0}=\left(0,0, v_{0}\right)$ directed along the $z$-axis; thus the equilibrium mass flow is not field-aligned. No mass flow is present outside the tube, therefore the surrounding photospheric medium is considered to be uniformly magnetised ( $B_{\mathrm{e}} \hat{z}$ is constant), uniform ( $\rho_{\mathrm{e}}$ constant $)$, and lacking mass flow at equilibrium.

In cylindrical equilibrium the magnetic field and plasma pressure satisfy the equilibrium condition in the radial direction

$\frac{\mathrm{d}}{\mathrm{d} r}\left(p_{\mathrm{i}}+\frac{B_{\mathrm{i}}^{2}}{2 \mu}\right)=-\frac{B_{\mathrm{i} \phi}^{2}}{\mu r}$

Here, $B_{\mathrm{i}}(r)=\left(B_{\mathrm{i} \phi}^{2}+B_{\mathrm{i} z}^{2}\right)^{1 / 2}=\left|\boldsymbol{B}_{\mathrm{i}}\right|$ denotes the strength of the equilibrium magnetic field, and $\mu$ is the magnetic permeability. We note that in Eq. (1) the total (plasma plus magnetic) pressure gradient is balanced by the tension force (the righthand side of Eq. (1)) in the twisted field. We consider the special case of an equilibrium with uniform twist, i.e., the one for which $B_{\mathrm{i} \phi}(r) / r B_{\mathrm{iz}}(r)$ is a constant. Accordingly, the background magnetic field is assumed to be

$\boldsymbol{B}(r)= \begin{cases}\left(0, A r, B_{\mathrm{i} z}\right) & \text { for } r \leqslant a, \\ \left(0,0, B_{\mathrm{e}}\right) & \text { for } r>a,\end{cases}$

where $A, B_{\mathrm{i} z}$, and $B_{\mathrm{e}}$ are constant. Then the equilibrium condition (1) gives the equilibrium plasma pressure $p_{\mathrm{i}}(r)$ as

$p_{\mathrm{i}}(r)=p_{0}-\frac{A^{2} r^{2}}{\mu}$

where $p_{0}$ is the plasma pressure at the centre of the tube. We note that the jump in the value of $B_{\phi}(r)$ across $r=a$ implies a surface current there.

The plasma motion is governed by the set of linearised MHD equations for an ideal incompressible plasma:

$$
\begin{aligned}
& \rho \frac{\partial}{\partial t} \boldsymbol{v}_{1}+\rho\left(\boldsymbol{v}_{0} \cdot \nabla\right) \boldsymbol{v}_{1}+\nabla\left(p_{1}+\frac{\boldsymbol{B}_{0} \cdot \boldsymbol{B}_{1}}{\mu}\right) \\
& -\frac{1}{\mu}\left(\boldsymbol{B}_{0} \cdot \nabla\right) \boldsymbol{B}_{1}-\frac{1}{\mu}\left(\boldsymbol{B}_{1} \cdot \nabla\right) \boldsymbol{B}_{0}=0, \\
& \frac{\partial}{\partial t} \boldsymbol{B}_{1}-\nabla \times\left(\boldsymbol{v}_{1} \times \boldsymbol{B}_{0}\right)-\nabla \times\left(\boldsymbol{v}_{0} \times \boldsymbol{B}_{1}\right)=0, \\
& \nabla \cdot \boldsymbol{v}_{1}=0, \\
& \nabla \cdot \boldsymbol{B}_{1}=0 .
\end{aligned}
$$

Here, the index " 0 " denotes equilibrium values of the fluid velocity and the medium magnetic field, and the index "1" their perturbations. Below, the sum $p_{1}+\boldsymbol{B}_{0} \cdot \boldsymbol{B}_{1} / \mu$ in Eq. (3) will be replaced by $p_{1 \text { tot }}$, which represents the total pressure perturbation.

Assuming that all perturbations are proportional to $g(r) \exp \left[\mathrm{i}\left(-\omega t+m \phi+k_{z} z\right)\right]$ with $g(r)$ being just a function of $r$, and that in cylindrical coordinates the nabla operator has the form

$\nabla \equiv \frac{\partial}{\partial r} \hat{r}+\frac{1}{r} \frac{\partial}{\partial \phi} \hat{\phi}+\frac{\partial}{\partial z} \hat{z}$

from the above set of equations one can obtain a second-order differential equation for the total pressure perturbation $p_{1 \text { tot }}$

$\left[\frac{\mathrm{d}^{2}}{\mathrm{~d} r^{2}}+\frac{1}{r} \frac{\mathrm{d}}{\mathrm{d} r}-\left(\kappa^{2}+\frac{m^{2}}{r^{2}}\right)\right] p_{1 \text { tot }}=0$,

as well as an expression for the radial component $v_{1 r}$ of the fluid velocity perturbation $v_{1}$ in terms of $p_{1 \text { tot }}$ and its first derivative

$v_{1 r}=-\mathrm{i} \frac{1}{\rho} \frac{1}{Y} \frac{\omega-\boldsymbol{k} \cdot \boldsymbol{v}_{0}}{\left(\omega-\boldsymbol{k} \cdot \boldsymbol{v}_{0}\right)^{2}-\omega_{\mathrm{A}}^{2}}\left(\frac{\mathrm{d}}{\mathrm{d} r} p_{1 \text { tot }}-Z \frac{m}{r} p_{1 \text { tot }}\right)$.

In Eq. (7), $\kappa$ is the so-called wave attenuation coefficient, which characterises the space structure of the wave and whose squared magnitude is given by the expression

$\kappa^{2}=k_{z}^{2}\left(1-\frac{4 A^{2} \omega_{\mathrm{A}}^{2}}{\mu \rho\left[\left(\omega-\boldsymbol{k} \cdot \boldsymbol{v}_{0}\right)^{2}-\omega_{\mathrm{A}}^{2}\right]^{2}}\right)$, 
where

$\omega_{\mathrm{A}}=\frac{1}{\sqrt{\mu \rho}}\left(m A+k_{z} B_{z}\right)$

is the so-called local Alfvén frequency (Bennett et al. 1999). The numerical coefficients $Z$ and $Y$ in the expression of $v_{1 r}$ (see Eq. (8)) are respectively

$Z=\frac{2 A \omega_{\mathrm{A}}}{\sqrt{\mu \rho}\left[\left(\omega-\boldsymbol{k} \cdot \boldsymbol{v}_{0}\right)^{2}-\omega_{\mathrm{A}}^{2}\right]} \quad$ and $\quad Y=1-Z^{2}$.

As seen from the expressions for the wave attenuation coefficient and the radial fluid velocity component perturbation, the two quantities have different values inside and outside the twisted flux tube owing to the different spatial structure of the magnetic field in both media, and to the different mass densities ( $\rho_{\mathrm{i}}$ and $\rho_{\mathrm{e}}$, respectively). It is important to note that the attenuation coefficient $\kappa_{\mathrm{e}}$ outside the tube is simply equal to $k_{z}$, whilst that inside the tube can become a purely imaginary quantity, thus allowing the propagation of pseudosurface (body) waves along the tube. Notably the twisted magnetic field inside the tube enables the existence of these waves, which otherwise are pure surface modes (Bennett et al. 1999). the jet

Equation (7) has two different solutions inside and outside $p_{1 \text { tot }}(r)= \begin{cases}\alpha_{\mathrm{i}} I_{\mathrm{m}}\left(\kappa_{\mathrm{i}} r\right) & \text { for } r \leqslant a, \\ \alpha_{\mathrm{e}} K_{\mathrm{m}}\left(k_{z} r\right) & \text { for } r>a .\end{cases}$

Here $I_{\mathrm{m}}$ and $K_{\mathrm{m}}$ are the modified Bessel functions of the first and second kind, and $\alpha_{\mathrm{i}}$ and $\alpha_{\mathrm{e}}$ are arbitrary constants.

The boundary conditions have to ensure that the normal component of the interface perturbation

$\xi_{r}=-\frac{v_{1 r}}{\mathrm{i}\left(\omega-\boldsymbol{k} \cdot \boldsymbol{v}_{0}\right)}$

remains continuous across the unperturbed tube boundary $r=a$, and also that the total Lagrangian pressure is conserved across the perturbed boundary. This leads to the conditions (Bennett et al. 1999)

$\left[\xi_{r}\right]^{\text {inner }}=\left[\xi_{r}\right]^{\text {outer }}$,

$\left[p_{1 \text { tot }}-\frac{B_{\phi}^{2}}{\mu a} \xi_{r}\right]^{\text {inner }}=\left[p_{1 \text { tot }}-\frac{B_{\phi}^{2}}{\mu a} \xi_{r}\right]^{\text {outer }}$.

Applying boundary conditions (11) and (12) to our solutions of $p_{1 \text { tot }}$ and $v_{1 r}$ (and respectively $\xi_{r}$ ), we obtain after some algebra the dispersion relation of the normal modes propagating along a twisted magnetic tube with axial mass flow $\boldsymbol{v}_{0}$

$$
\begin{array}{r}
\frac{\left[\left(\omega-\boldsymbol{k} \cdot \boldsymbol{v}_{0}\right)^{2}-\omega_{\mathrm{Ai}}^{2}\right] \frac{\kappa_{\mathrm{i}} a I_{\mathrm{m}}^{\prime}\left(\kappa_{\mathrm{i}} a\right)}{I_{\mathrm{m}}\left(\kappa_{\mathrm{i}} a\right)}-2 m \omega_{\mathrm{Ai}} \frac{A}{\sqrt{\mu \rho_{\mathrm{i}}}}}{\left[\left(\omega-\boldsymbol{k} \cdot \boldsymbol{v}_{0}\right)^{2}-\omega_{\mathrm{Ai}}^{2}\right]^{2}-4 \omega_{\mathrm{Ai}}^{2} \frac{A^{2}}{\mu \rho_{\mathrm{i}}}} \\
=\frac{\frac{k_{z} a K_{\mathrm{m}}^{\prime}\left(k_{z} a\right)}{K_{\mathrm{m}}\left(k_{z} a\right)}}{\frac{\rho_{\mathrm{e}}}{\rho_{\mathrm{i}}}\left(\omega^{2}-\omega_{\mathrm{Ae}}^{2}\right)+\frac{A^{2}}{\mu \rho_{\mathrm{i}}} \frac{k_{z} a K_{\mathrm{m}}^{\prime}\left(k_{z} a\right)}{K_{\mathrm{m}}\left(k_{z} a\right)}} .
\end{array}
$$

This dispersion relation is a generalisation of Eq. (23) in the work by Bennett et al. (1999) that is applicable to a twisted magnetic flux tube without flow and of Eq. (13) in the work by Zaqarashvili et al. (2010) that is applicable to a twisted magnetic flux tube with a flow embedded in a non-magnetic environment. As we have already seen, the wave frequency $\omega$ is Doppler-shifted inside the jet. In the above equation a prime ( () denotes the derivative of a Bessel function to its dimensionless argument, and the local Alfvén frequencies inside and outside the tube are correspondingly

$\omega_{\mathrm{Ai}}=\frac{1}{\sqrt{\mu \rho_{\mathrm{i}}}}\left(m A+k_{z} B_{\mathrm{iz}}\right) \quad$ and $\quad \omega_{\mathrm{Ae}}=\frac{k_{z} B_{\mathrm{e}}}{\sqrt{\mu \rho_{\mathrm{e}}}}$.

The wave attenuation coefficient inside the tube, according to Eq. (9), is given by

$\kappa_{\mathrm{i}}=k_{z}\left(1-\frac{4 A^{2} \omega_{\mathrm{Ai}}^{2}}{\mu \rho_{\mathrm{i}}\left[\left(\omega-\boldsymbol{k} \cdot \boldsymbol{v}_{0}\right)^{2}-\omega_{\mathrm{Ai}}^{2}\right]^{2}}\right)^{1 / 2}$.

Assuming no twist, when $B_{\mathrm{i} \phi}=0$, the dispersion relation (13) reduces to

$$
\begin{aligned}
\frac{\rho_{\mathrm{e}}}{\rho_{\mathrm{i}}}\left(\omega^{2}-\right. & \left.k_{z}^{2} v_{\mathrm{Ae}}^{2}\right) \frac{I_{\mathrm{m}}^{\prime}\left(k_{z} a\right)}{I_{\mathrm{m}}\left(k_{z} a\right)} \\
- & {\left[\left(\omega-\boldsymbol{k} \cdot \boldsymbol{v}_{0}\right)^{2}-k_{z}^{2} v_{\mathrm{Ai}}^{2}\right] \frac{K_{\mathrm{m}}^{\prime}\left(k_{z} a\right)}{K_{\mathrm{m}}\left(k_{z} a\right)}=0 . }
\end{aligned}
$$

The Alfvén speeds in this equation are expressed in terms of the background magnetic fields in both media and the corresponding mass densities via the well-known definitions

$v_{\mathrm{Ai}}=\frac{B_{\mathrm{i}}}{\sqrt{\mu \rho_{\mathrm{i}}}} \quad$ and $\quad v_{\mathrm{Ae}}=\frac{B_{\mathrm{e}}}{\sqrt{\mu \rho_{\mathrm{e}}}}$,

respectively. Equation (14) is akin to a dispersion relation in the same form as was previously obtained by Edwin \& Roberts (1983) for untwisted magnetic tubes without flow. We also recall that for the kink mode $(m=1)$ one defines the so-called kink speed (Edwin \& Roberts 1983)

$c_{\mathrm{k}}=\left(\frac{\rho_{\mathrm{i}} v_{\mathrm{Ai}}^{2}+\rho_{\mathrm{e}} v_{\mathrm{Ae}}^{2}}{\rho_{\mathrm{i}}+\rho_{\mathrm{e}}}\right)^{1 / 2}=\left(\frac{v_{\mathrm{Ai}}^{2}+\left(\rho_{\mathrm{e}} / \rho_{\mathrm{i}}\right) v_{\mathrm{Ae}}^{2}}{1+\rho_{\mathrm{e}} / \rho_{\mathrm{i}}}\right)^{1 / 2}$,

which characterises the propagation of transverse perturbations.

\section{Numerical solutions and wave dispersion diagrams}

We focus our study on the propagation of the kink mode, i.e., for $m=1$. It is obvious that Eqs. (13) and (14) can be solved only numerically. The first step is to define the input parameters that characterise the twisted magnetic tube and the axial flow, and to normalise all variables in the dispersion equations. The density contrast between the tube and its environment is characterised by the parameter $\eta=\rho_{\mathrm{e}} / \rho_{\mathrm{i}}$. In investigating the kink instability in twisted magnetic tubes with axial and field-aligned flows with their equilibrium magnetic fields in the form $\boldsymbol{B}(r)=\left(0, A r, B_{0}\right)$, Zaqarashvili et al. (2010) and Díaz et al. (2011) chose as twist characteristics the so-called "dimensionless pitch" $k_{z} p$, in which the specific length $p$ is defined (in their notation) as

$p=\frac{B_{0}}{A} \quad$ or more generally as $\quad p=\frac{B_{z}}{A}$.

That dimensionless pitch can be presented in the form $k_{z} p=$ $2 \pi p / \lambda$, where $2 \pi p$ is the magnetic field pitch in its common 
sense. As we showed, this parameter is associated with a fixed wavelength $\lambda$. To study the Kelvin-Helmholtz instability in these tubes we prefer to specify the twist by the ratio of the two magnetic field components, $B_{\phi}$ and $B_{z}$, evaluated at the inner boundary of the tube, $r=a$, i.e., via $B_{\phi} / B_{z}$, where $B_{\phi}=A a$. This choice is symbolically equivalent to the replacement of the wavelength $\lambda$ by the tube radius $a$. Following Aschwanden (2005), the magnetic twist parameter $B_{\phi} / B_{z}$ can be estimated as $B_{\phi} / B_{z}=\tan \theta$, where $\theta$ is the shear angle between the untwisted and twisted field line. The geometric shear angle, $\theta$, can observationally be measured in twisted photospheric magnetic tubes and in twisted coronal loops. We note that our choice of the twist parameter allows us to immediately compare the dispersion curves of kink waves and the growth rates when the waves become unstable in twisted magnetic tubes with those in untwisted ones.

As usual, we normalise the velocities to the Alfvén speed $v_{\mathrm{Ai}}=B_{\mathrm{i}} /\left(\mu \rho_{\mathrm{i}}\right)^{1 / 2}$, where $B_{\mathrm{i}}=\left(B_{\mathrm{i} \phi}^{2}+B_{\mathrm{i} z}^{2}\right)^{1 / 2}$ is evaluated at the inner boundary of the tube, $r=a$. Thus, we introduce the dimensionless wave phase velocity $V_{\mathrm{ph}}=v_{\mathrm{ph}} / v_{\mathrm{Ai}}$ and the AlfvénMach number $M_{\mathrm{A}}=v_{0} / v_{\mathrm{Ai}}$, the latter characterising the axial mass flow. The wavelength is normalised to the tube radius $a$, which means that the dimensionless wave number is $K=k_{z} a$. It is worth to discuss the normalisation of the local Alfvén frequency $\omega_{\mathrm{Ai}}$. After multiplying both sides of Eq. (10) by the tube radius $a$ and extracting a $B_{\mathrm{i}}$ from the brackets, we derive that

$a \omega_{\mathrm{Ai}}=\frac{B_{\mathrm{i}}}{\sqrt{\mu \rho_{\mathrm{i}}}}\left(m \frac{B_{\mathrm{i} \phi}}{B_{\mathrm{i}}}+k_{z} a \frac{B_{\mathrm{i} z}}{B_{\mathrm{i}}}\right), \quad$ where $\quad \frac{B_{\mathrm{i} z}}{B_{\mathrm{i}}}=\left(1-\frac{B_{\mathrm{i} \phi}^{2}}{B_{\mathrm{i}}^{2}}\right)^{1 / 2}$.

To simplify the numerical code, and largely for clarity, we can redefine our twist parameter as $B_{\mathrm{i} \phi} / B_{\mathrm{i}} \equiv \varepsilon$, and consequently the normalised local Alfvén frequency is expressed in terms of the Alfvén speed $v_{\mathrm{Ai}}$, the dimensionless wave number $K$, and $\varepsilon$ :

$a \omega_{\mathrm{Ai}}=v_{\mathrm{Ai}}\left(m \varepsilon+K b_{z}\right)$,

where the dimensionless axial magnetic field component $b_{z}=$ $\left(1-\varepsilon^{2}\right)^{1 / 2}$. The new twist parameter, $\varepsilon$, is related to the real twist $B_{\phi} / B_{z}$ via the simple relation

$\varepsilon=\frac{B_{\phi} / B_{z}}{\sqrt{1+\left(B_{\phi} / B_{z}\right)^{2}}}$

Thus, if we have an observationally measured shear angle $\theta$ of some solar twisted magnetic tube, then $B_{\phi} / B_{z}=\tan \theta$ can be inserted into expression (16) to obtain the adopted twist $\varepsilon$ of that specific magnetic tube.

Before starting the numerical calculation, we have to specify the values of the input parameters. First we will examine, as in the work of Zaqarashvili et al. (2010), the case of an isolated twisted magnetic tube with axial mass flow, i.e., with $B_{\mathrm{e}}=0$, and hence for parameter $b \equiv B_{\mathrm{e}} / B_{\mathrm{i}}=0$. Then, we will study the influence of the external magnetic field on the instability onset by choosing $b$ equal to 0.1 and 0.5 , respectively. Concerning the density contrast, $\eta$, we consider two cases, namely $\eta=2$ and $\eta=0.1$. Following Ruderman (2007), to satisfy the ShafranovKruskal stability criterion for a kink instability, we assume that the azimuthal component of the magnetic field is smaller than its axial component, i.e., we will choose our twist parameter $\varepsilon$ to be always less than 1 . In particular, we will study the dispersion diagrams of kink waves and their growth rates (when the waves are unstable) for three fixed values of $\varepsilon$ : 0 (untwisted magnetic tube), 0.1, and 0.4. The Alfvén-Mach number $M_{\mathrm{A}}$ will take values from zero (no flow) to any reasonable number.

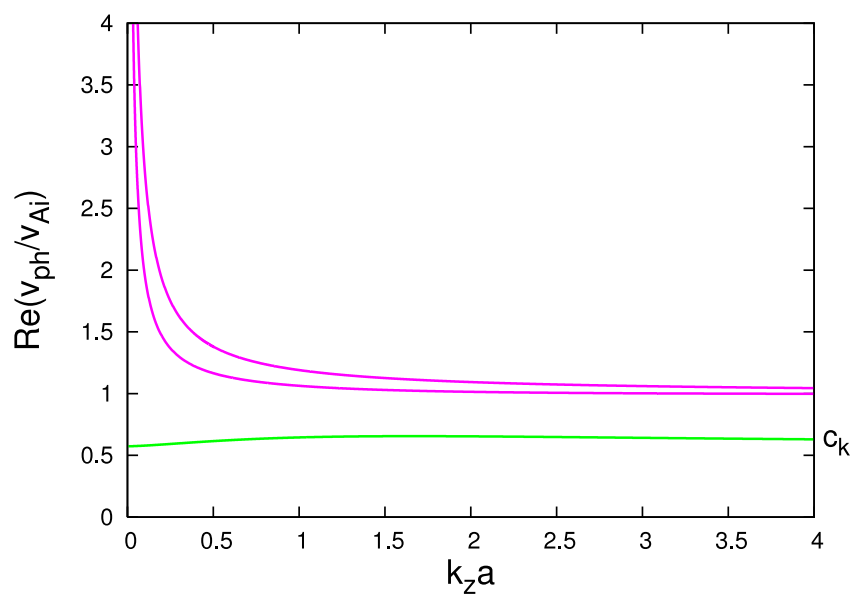

Fig. 1. Dispersion curves of kink waves propagating along a twisted magnetic flux tube at $\eta=2, B_{\mathrm{e}} / B_{\mathrm{i}}=0, B_{\mathrm{i} \phi} / B_{\mathrm{i}}=0.1$, and $M_{\mathrm{A}}=0$. The green curve is the dispersion curve of the wave associated with the kink speed $c_{\mathrm{k}}$. The two purple curves depict a band that contains nine dispersion curves of similar form, not plotted here.

\subsection{Kink waves in twisted magnetic tubes with a density contrast $\eta=2$}

We start by calculating the dispersion curves of kink waves assuming that the twist parameter $\varepsilon=0.1$, and that the angular wave frequency $\omega$ is real. As a reference, we first assume that the plasma in the flux tube is static, i.e., $M_{\mathrm{A}}=0$. The dispersion curves, which present the dependence of the normalised wave phase velocity, $v_{\mathrm{ph}} / v_{\mathrm{Ai}}$, on the normalised wave number, $k_{z} a$, are in this case shown in Fig. 1. One can recognise two types of waves: a sub-Alfvénic wave labelled $c_{k}$ (the green curve), and a family of super-Alfvénic waves accommodated in a narrow band depicted by the two purple dispersion curves. The green dispersion curve is related to the kink speed defined by Eq. (15). For a weakly twisted magnetic tube $(\varepsilon=0.1)$ with a density contrast $\eta=2$ the kink speed is equal to $0.5774 v_{\mathrm{Ai}}$ - our numerical code yields in the long wavelength limit, $c_{\mathrm{k}}=0.5738 v_{\mathrm{Ai}}$, which agrees well with the value calculated from Eq. (15). If we assume that the Alfvén speed inside the tube is typically $10 \mathrm{~km} \mathrm{~s}^{-1}$, then the kink speed in the twisted magnetic tube is equal to $5.7 \mathrm{~km} \mathrm{~s}^{-1}$, or approximately to $6 \mathrm{~km} \mathrm{~s}^{-1}$. We recall that the expression of $c_{\mathrm{k}}$ was defined for untwisted magnetic tubes and there is no guarantee that it will be valid for twisted tubes as well.

Bennett et al. (1999), in studying the kink wave propagation in a twisted magnetic tube with parameters similar to ours, had discovered a band that contains an infinite number of pseudosurface (body) wave harmonics (Fig. 5a in their paper). Among those, generally super-Alfvénic, waves we were able to easily calculate only 11 dispersion curves from Eq. (13) with search steps (in decreasing order) from 0.00025 to 0.000000025 . They are not plotted in Fig. 1 because there is no room for all of them in that narrow band, but they will be shown shortly in a similar plot for a tube with $\varepsilon=0.4$. With diminishing search step, taking it equal to 0.0000000025 or smaller, one can extract a few (3) additional curves, but finding them is an extremely hard numerical task - for such small search steps the data are very noisy. However, these high-harmonics pseudosurface kink waves are not too important to us since we are primarily interested in the evolution of the kink mode associated with the kink speed $c_{\mathrm{k}}$ when the plasma starts to flow. It is worth noticing that the $\mathrm{c}_{\mathrm{k}}$-labelled dispersion curve for small dimensionless wave numbers, till $k_{z} a=0.1228$, describes a pseudosurface (body) wave, 

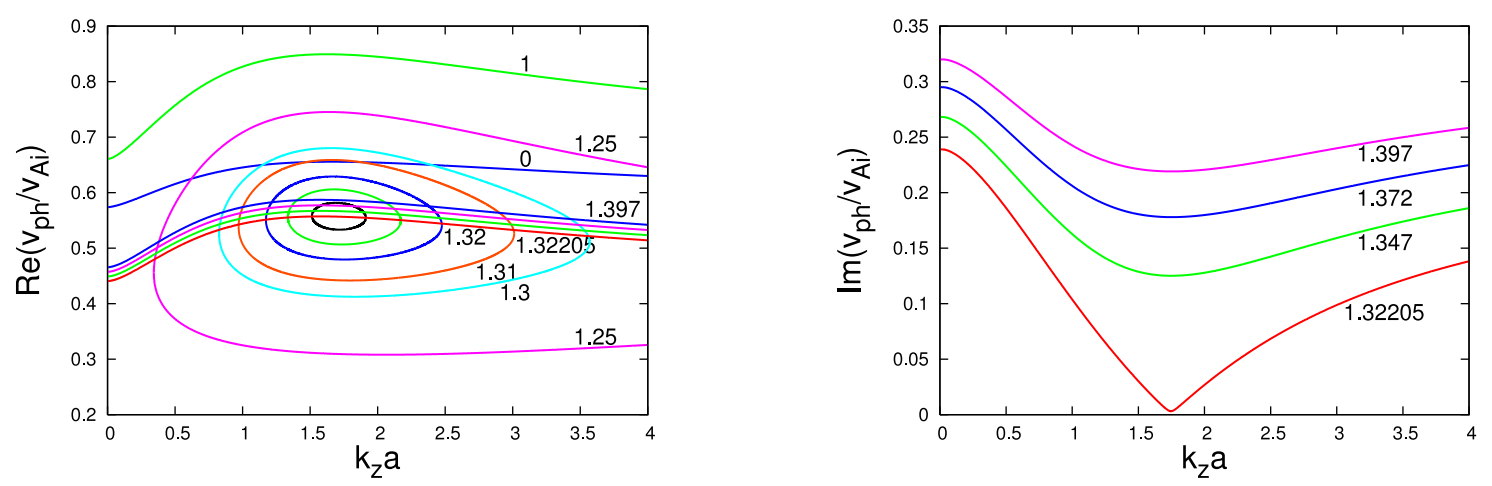

Fig. 2. (Left panel) Dispersion curves of forward propagating stable and unstable kink waves in a twisted magnetic flux tube with the same input parameters as in Fig. 1 for various values of the Alfvén-Mach number $M_{\mathrm{A}}$. The non-labelled green closed curve corresponds to $M_{\mathrm{A}}=1.325$, and the black one - to $M_{\mathrm{A}}=1.328$. (Right panel) Growth rates of the unstable kink waves for $M_{\mathrm{A}}=1.32205,1.347,1.372$, and 1.397 .
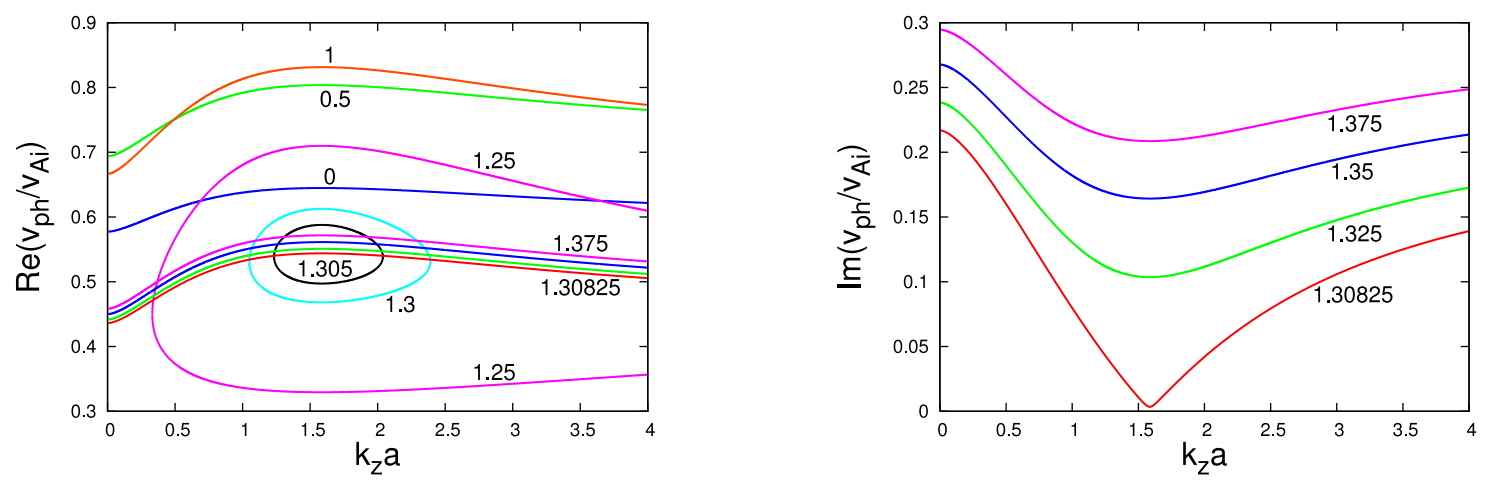

Fig. 3. (Left panel) Dispersion curves of forward propagating stable and unstable kink waves in an untwisted magnetic flux tube at $\eta=2$ and $B_{\mathrm{e}} / B_{\mathrm{i}}=0$ for various values of the Alfvén-Mach number $M_{\mathrm{A}}$. (Right panel) Growth rates of the unstable kink waves for $M_{\mathrm{A}}=1.30825,1.325,1.35$, and 1.375 .

while behind that value the wave is a pure surface mode. These types of waves are sometimes called hybrid waves (Bennett et al. 1999).

It is essential to emphasise that the dispersion Eq. (13) also yields solutions for negative values of the wave phase velocity (i.e., for backward-propagating waves), which are a mirror image of the solutions in the positive semi-space. Including the flow, the whole pattern shifts en bloc upwards, changing of course both the $c_{k}$-labelled dispersion curve and the wave harmonics dispersion curves. For each $M_{\mathrm{A}}$ we have two $c_{\mathrm{k}}$-dispersion curves that initially, for small Alfvén-Mach numbers, are independent, but at certain magnitudes of $M_{\mathrm{A}}$ begin to interact; for example, they form semi-closed or closed dispersion curves by merging. These dispersion curves are an indication that we are in a region in which the kink wave may become unstable (see Zhelyazkov 2010).

To study the stability/instability status of the $c_{\mathrm{k}}$-kink wave, we have to assume that the wave frequency is complex, i.e., $\omega \rightarrow$ $\omega+\mathrm{i} \gamma$, where $\gamma$ is the expected instability growth rate. Thus, the dispersion equation becomes complex (complex wave phase velocity and real wave number) and deriving the solutions to a transcendental complex equation is generally a hard task (see Acton 1990). We numerically solve Eq. (13) using the Muller method (Muller 1956) to find the complex roots at fixed input parameters $\eta=2, b=0$, and $\varepsilon=0.1$, and varying Alfvén-Mach numbers $M_{\mathrm{A}}$ from zero to generally reasonable values. The evolution of the two kink waves with increasing flow velocity (or equivalently $M_{\mathrm{A}}$ ) is shown in Fig. 2 . We note that while for rest plasma or small Alfvén-Mach numbers the lower kink wave (i.e., the one that is a mirror image of the green curve in Fig. 1) is a backward one, for $M_{\mathrm{A}}=1$ it becomes a forward wave. At the next increase of the Alfvén-Mach number the two dispersion curves change their form and structure - initially being hybrid modes, for $M_{\mathrm{A}}>1.23$ they are pure surface waves. The most interesting observation is that for $M_{\mathrm{A}} \geqslant 1.2173$ both curves begin to merge and for $M_{\mathrm{A}} \geqslant 1.2933$ they form a closed dispersion curve. The ever increasing $M_{\mathrm{A}}$ yields yet smaller closed dispersion curves the smallest one depicted in the left panel of Fig. 2 corresponds to $M_{\mathrm{A}}=1.328$. All these dispersion curves present a stable propagation of the kink waves. However, for $M_{\mathrm{A}} \geqslant 1.32205$ we obtain a new family of wave dispersion curves that correspond to an unstable wave propagation. We plot in Fig. 2 four curves of that kind that were calculated for $M_{\mathrm{A}}=1.32205,1.347,1.372$, and 1.397 , respectively. The growth rates of the unstable waves are shown in the right panel of the same figure. The instability that arises is of the Kelvin-Helmholtz type. Note the shape of red curve labelled 1.32205: the growth rate has relatively high values for small normalised wave numbers, then passes through a minimum (its relative value there is $\sim 0.003$ ) after which it starts to grow. That specific curve is to some extent a marginal curve for values of $M_{\mathrm{A}}$ lower than 1.32205 the kink wave is stable whilst at $M_{\mathrm{A}} \geqslant 1.32205$ the wave is definitely unstable. This means that the instability onset at the critical $M_{\mathrm{A}}$ will prevent the occurrence of the neutral dispersion curve with $M_{\mathrm{A}}=1.328$.

It is interesting to see how the twist of the magnetic field has changed the dispersion curves and growth rates of unstable kink 
I. Zhelyazkov and T. V. Zaqarashvili: Kelvin-Helmholtz instability of kink waves in twisted magnetic tubes

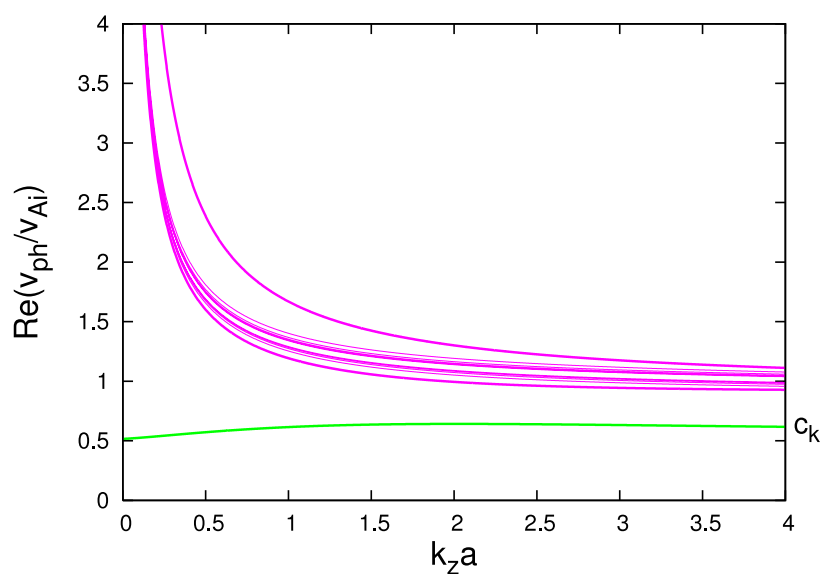

Fig. 4. Dispersion curves of kink waves propagating along a twisted magnetic flux tube at $\eta=2, B_{\mathrm{e}} / B_{\mathrm{i}}=0, B_{\mathrm{i} \phi} / B_{\mathrm{i}}=0.4$, and $M_{\mathrm{A}}=0$. The green curve is the dispersion curve of the wave associated with the kink speed $c_{\mathrm{k}}$. The purple curves depict all 11 dispersion curves; 9 of them are divided into two sub-groups. The "evolution" of those curves when increasing $k_{z} a$ can be seen in the next figure, which shows them near the left top and right bottom corners of the plot.

waves calculated for an untwisted magnetic tube. In that case the dispersion curves and growth rates can be obtained through the exact numerical solutions of Eq. (14) (see Zhelyazkov 2010). The results are shown in Fig. 3. Comparing Figs. 2 and 3, we immediately see that the dispersion diagrams and growth rates of the unstable kink waves are very similar. There are differences, of course; firstly the magnetic field twist visibly extends the closed dispersion curves in the horizontal direction, and secondly, it slightly increases the threshold Alfvén-Mach number for the onset of the Kelvin-Helmholtz instability - for the untwisted magnetic tube it is equal to 1.30825 , whilst for the twisted one it is slightly higher - the corresponding value is 1.32205 . The similarities between the dispersion diagrams and the growth rates are important to us - they indirectly confirm the correctness of our numerical solutions derived by solving the complex dispersion Eq. (13).

Figure 4 shows the dispersion curves of kink waves when the twist parameter $\varepsilon=0.4$ and there is no flow. Now one can see all 11 easily derived dispersion curves of the wave harmonics. They have similar shapes, and as seen in Figs. 4 and 5, one can distinguish two sub-groups of four and five curves, respectively. We note that the eleventh (the highest) dispersion curve is not visible in the top panel of Fig. 5 because it simply begins at about $k_{z} a=0.23$, which is far beyond the right side of the plot. Another important observation is that the kink speed is markedly lower than expected from the Eq. (15) value - here its normalised magnitude is equal to 0.5167 , which corresponds to a kink speed of $5 \mathrm{~km} \mathrm{~s}^{-1}$. The kink wave itself is a hybrid mode till $k_{z} a=0.5623$ it is a pseudosurface (body) wave, becoming a pure surface wave for the next values of the normalised wave number. When plasma in the tube is flowing, the kink waves are pure surface mode for $M_{\mathrm{A}}>1.197$.

The dispersion curves and growth rates of kink waves for this higher value of the magnetic field twist are given in Fig. 6. At the left panel of this figure we see dramatic changes in the shape and position of the closed dispersion curves. The most striking changes, however, are the circumstances that the onset of unstable kink waves, like in Fig. 2, starts at a value of the Alfvén-Mach number lower than that of the narrowest closed dispersion curve -1.250075 vs. 1.29 ! In untwisted flux tubes the
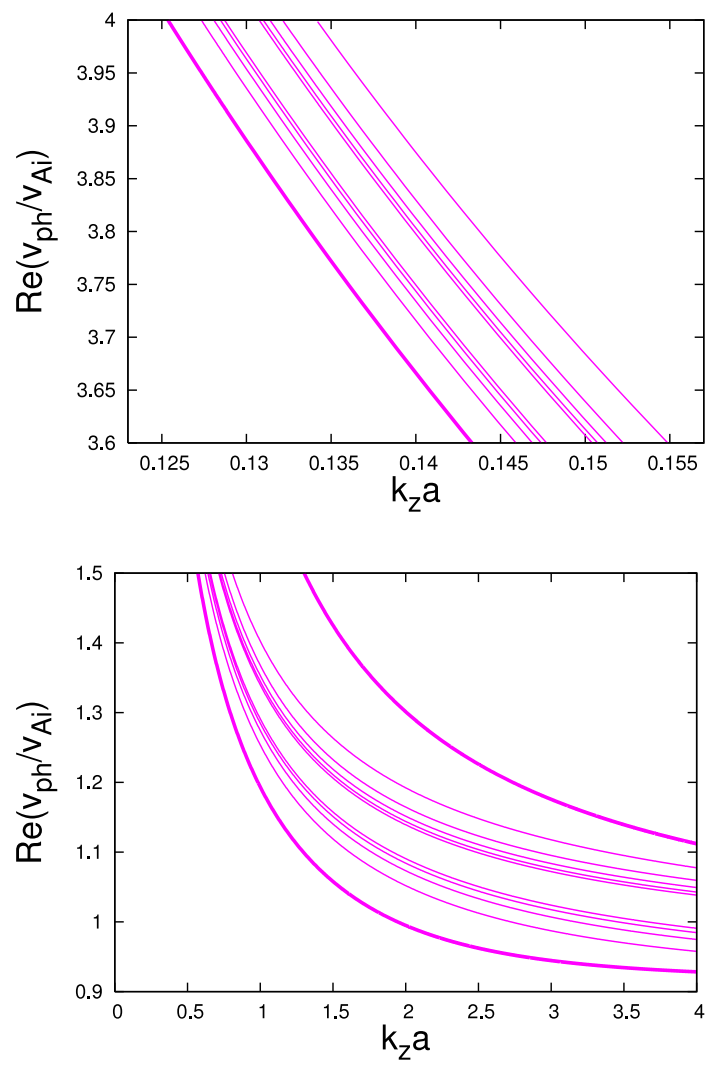

Fig. 5. Zoomed-in parts of the 11 dispersion curves near the left top (top panel) and right bottom (bottom panel) corners of the plot in Fig. 4.

kink mode appears at Alfvén-Mach numbers higher than those of the smallest closed dispersion curves, as seen in Fig. 3 (cf. also Fig. 4 in Zhelyazkov (2012a)). In reality, of course, the onset of a Kelvin-Helmholtz instability in a twisted tube will "reject" the co-existence of stable kink waves like those labelled 1.267, 1.28, 1.287, and 1.29 in Fig. 6.

Similar calculations were performed for the two external magnetic fields specified by $b=0.1$ and 0.5 . As expected, the environment's magnetic field stabilises the kink wave propagation (Bennett et al. 1999) - the instability onset occurs at slightly higher threshold Alvfén-Mach numbers, but the shapes of the dispersion and growth rate curves are similar to those of an isolated twisted flux tube as depicted in Figs. 2, 3, and 6. The influence of the magnetic field twist, $\varepsilon$, at a fixed external magnetic field, $b$, and that of the environment's magnetic field at a fixed tube twist on the specific growth rate curves are illustrated in Figs. 7 and 8. As seen in Fig. 7, at a fixed magnetic field configuration, the tube twist shifts to the right the minimum of the specific growth rate curve. This is most pronounced at $b=0.5$. A twist of 0.4 notably decreases the wave growth rate for each $b$ and yields the lowest threshold Alfvén-Mach number for a given $b$. Thus, for a photospheric twisted magnetic tube with $\varepsilon=0.4$ flow speeds in the range $12.5-13.6 \mathrm{~km} \mathrm{~s}^{-1}$ can ensure the occurrence of a Kelvin-Helmholtz instability of the kink waves propagating along that tube. These flow speeds can be observed/detected in the solar photosphere. It becomes clear from Fig. 8 that an external magnetic field visibly increases the threshold Alfvén-Mach number and diminishes the wave growth rate - the latter effect is strongest for a magnetic tube with twist parameter $\varepsilon=0.4$. 

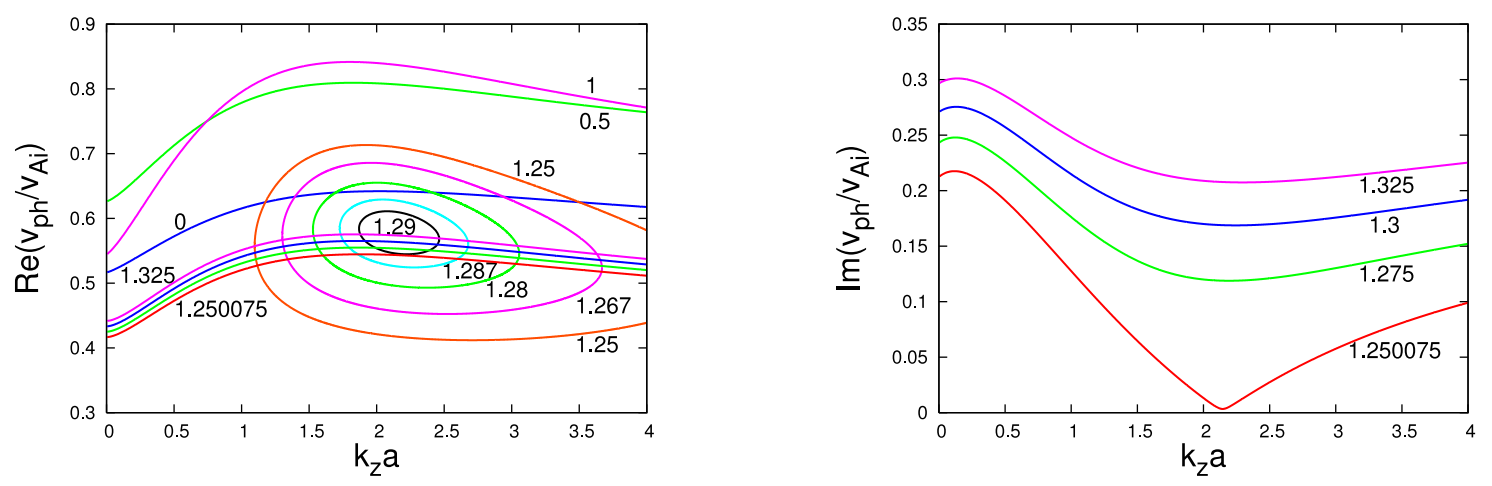

Fig. 6. (Left panel) Dispersion curves of forward-propagating stable and unstable kink waves in a twisted magnetic flux tube with the same input parameters as in Fig. 4 for various values of the Alfvén-Mach number $M_{\mathrm{A}}$. (Right panel) Growth rates of the unstable kink waves for $M_{\mathrm{A}}=1.250075,1.275,1.3$, and 1.325 .
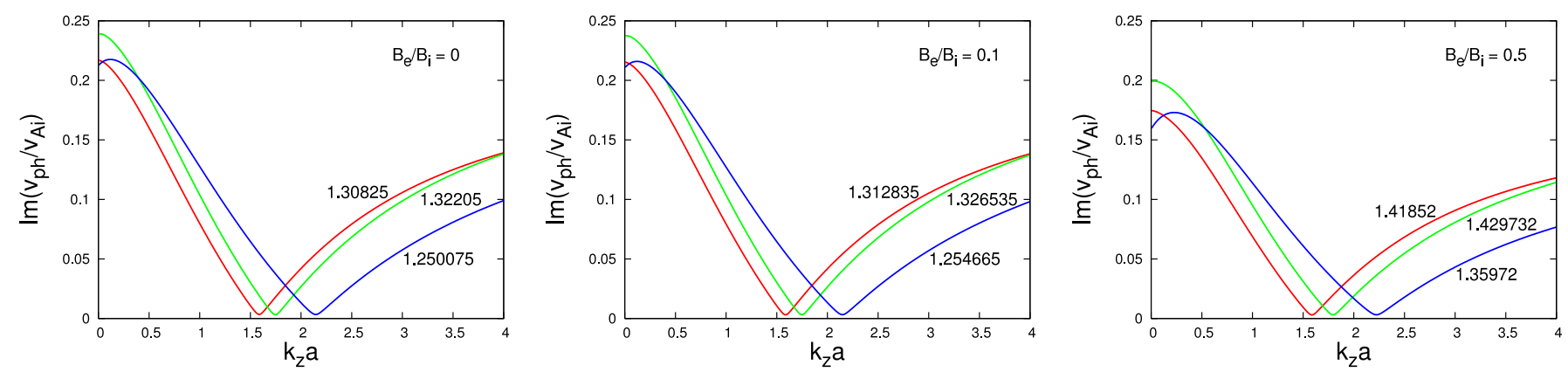

Fig. 7. Specific growth rates of kink waves propagating along an untwisted/twisted magnetic flux tube at $\eta=2, B_{\mathrm{e}} / B_{\mathrm{i}}=0($ left panel $), B_{\mathrm{e}} / B_{\mathrm{i}}=0.1$ (middle panel), and $B_{\mathrm{e}} / B_{\mathrm{i}}=0.5$ (right panel) for various values of the twist parameter $B_{\mathrm{i} \phi} / B_{\mathrm{i}}$, equal to 0 (red curve), 0.1 (green curve), and 0.4 (blue curve), respectively. The curve labels denote the threshold Alfvén-Mach number.
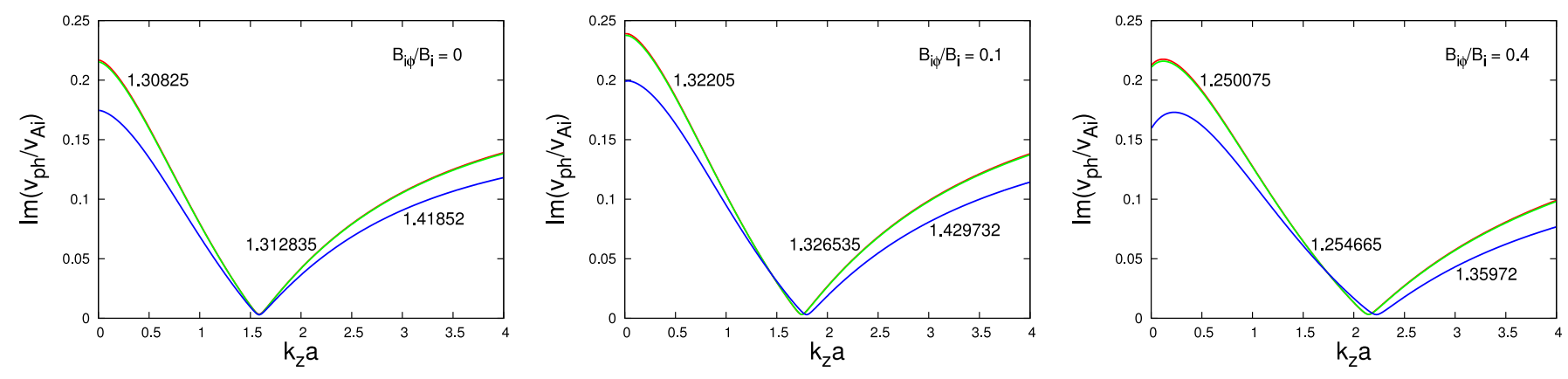

Fig. 8. Specific growth rates of kink waves propagating along an untwisted/twisted magnetic flux tube at $\eta=2, B_{\mathrm{i} \phi} / B_{\mathrm{i}}=0\left(\right.$ left panel), $B_{\mathrm{i} \phi} / B_{\mathrm{i}}=0.1$ (middle panel), and $B_{\mathrm{i} \phi} / B_{\mathrm{i}}=0.4$ (right panel) for various values of the environment's magnetic field $B_{\mathrm{e}} / B_{\mathrm{i}}$, equal to 0 (red curve), 0.1 (green curve), and 0.5 (blue curve), respectively. The curve labels denote the threshold Alfvén-Mach number.

\subsection{Kink waves in twisted magnetic tubes with a density contrast $\eta=0.1$}

A density contrast $\eta=0.1$ is perhaps more suitable for a coronal and upper chromospheric magnetic flux tube than to a photospheric one, but nevertheless, studying this case is important for us to see how the density contrast affects the threshold/critical Alfvén-Mach numbers for the onset of the Kelvin-Helmholtz instability of kink waves propagating along twisted magnetic tubes. The procedure for performing calculations and plotting dispersion diagrams is the same as in the previous subsection. This lower value of $\eta$ yields markedly higher threshold AlfvénMach numbers as well as higher growth rates compared to $\eta=2$.
Here, in Fig. 9, we show only one set of plots - namely that for an isolated flux tube $(b=0)$ with a twist of 0.4 . At these values of the input parameters we obtain the smallest threshold $M_{\mathrm{A}}$. In Fig. 10, like in Fig. 7, we present the specific growth rate curves at fixed external magnetic fields for the three different values of the magnetic twist. As seen from this figure, for that value of the density contrast $(\eta=0.1)$ the influence of the magnetic twist, $\varepsilon$, on the shapes and positions of the specific growth rate curves, irrespective of the magnitude of the environment's magnetic field, is negligibly small. That is why a figure, similar to Fig. 8, is useless - in each subfigure for given $\varepsilon$ the three curves would practically coincide because the differences are only in the second/third places behind the decimal point. As for a photospheric 
I. Zhelyazkov and T. V. Zaqarashvili: Kelvin-Helmholtz instability of kink waves in twisted magnetic tubes
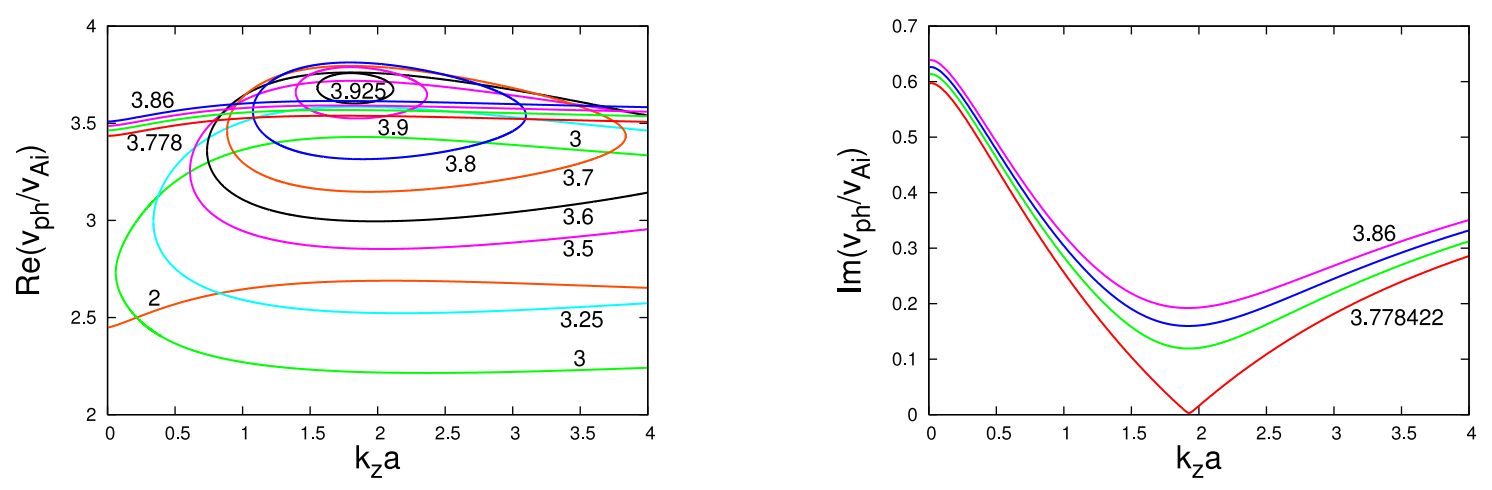

Fig. 9. (Left panel) Dispersion curves of forward-propagating stable and unstable kink waves in a twisted magnetic flux tube with $\eta=0.1$, $B_{\mathrm{e}} / B_{\mathrm{i}}=0$, and $B_{\mathrm{i} \phi} / B_{\mathrm{i}}=0.4$ for various values of the Alfvén-Mach number $M_{\mathrm{A}}$. (Right panel) Growth rates of the unstable kink waves for $M_{\mathrm{A}}=3.778422,3.81,3.835$, and 3.86 .
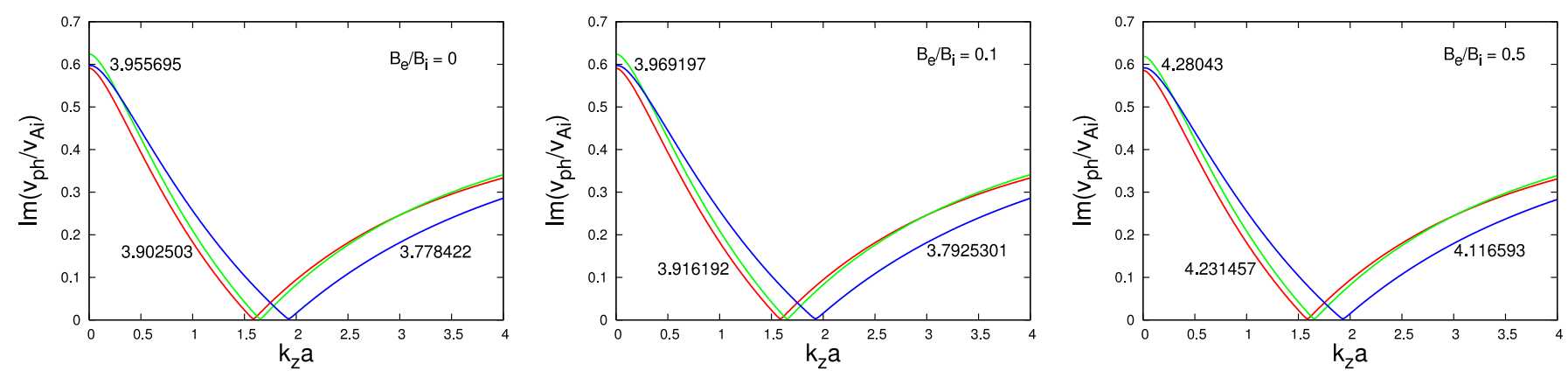

Fig. 10. Specific growth rates of kink waves propagating along an untwisted/twisted magnetic flux tube at $\eta=0.1, B_{\mathrm{e}} / B_{\mathrm{i}}=0$ (left panel), $B_{\mathrm{e}} / B_{\mathrm{i}}=0.1$ (middle panel), and $B_{\mathrm{e}} / B_{\mathrm{i}}=0.5$ (right panel) for various values of the twist parameter $B_{\mathrm{i} \phi} / B_{\mathrm{i}}$, equal to 0 (red curve), 0.1 (green curve), and 0.4 (blue curve), respectively. The curve labels denote the threshold Alfvén-Mach number.

twisted tube, one obtains the lowest threshold $M_{\mathrm{A}}$ at $b=0$ and $\varepsilon=0.4-$ the corresponding value is 3.778422 . If we accept that such a twisted magnetic tube can model a coronal loop, to estimate the Alfvén speed inside the tube, we assume a typical particle number density $n_{\mathrm{i}} \approx 10^{9} \mathrm{~cm}^{-3}$ (see Ugarte-Urra et al. 2005) and a magnetic field strength $B_{\mathrm{i}}=11 \mathrm{G}$ (see Verwichte et al. 2009); then $v_{\mathrm{Ai}} \approx 900 \mathrm{~km} \mathrm{~s}^{-1}$. With this high Alfvén speed one would need a flow speed of about $3400 \mathrm{~km} \mathrm{~s}^{-1}$ for the onset of an instability of the Kelvin-Helmholtz type. Since the detected flow velocities in the coronal loops are of the order of a few tens of kilometers per second, we can conclude that the kink waves in coronal loops with axial mass flows are stable against the Kelvin-Helmholtz instability.

\section{Conclusion}

We have studied the dispersion properties and the stability of the MHD kink modes running along the length of photospheric/chromospheric uniformly twisted magnetic tubes with axial mass flows. These were modelled as straight cylindrical jets of ideal incompressible plasma surrounded by a rest unmagnetised/magnetised also incompressible fully ionized medium. The wave propagation was investigated in the context of standard ideal magnetohydrodynamics by using linearised equations for the perturbations of the basic quantities: pressure, fluid velocity, and wave magnetic field. The derived dispersion equation describes the propagation of the kink mode influenced by the twist of the equilibrium magnetic field $\boldsymbol{B}_{\mathrm{i}}$ and the presence of moving plasma. The twist itself is characterised by the ratio of the azimuthal, $B_{\mathrm{i} \phi}$, to the axial, $B_{\mathrm{i} z}$, background magnetic field components both evaluated at the inner surface of the twisted magnetic tube. To avoid competition from the so-called kink instability, which is largely caused by the twist of the equilibrium magnetic field, we chose the ratio $B_{\mathrm{i} \phi} / B_{\mathrm{i} z}$ to be less than 1 . For simplicity and clarity of the numerical code used for solving the wave dispersion relation (in complex variables) we used a safer twist parameter $\varepsilon$ defined as $B_{\mathrm{i} \phi} / B_{\mathrm{i}}$. Our $\varepsilon$ and the real twist $B_{\mathrm{i} \phi} / B_{\mathrm{i} z}$ have generally rather close magnitudes, because they are related by a simple expression. The streaming plasma is characterised by its velocity $\boldsymbol{v}_{0}$, which is directed along the axis of the twisted magnetic tube. An alternative and more convenient way of specifying the mass flow is by defining the AlfvénMach number: the ratio of the jet speed to the Alfvén speed inside the jet, $M_{\mathrm{A}}=v_{0} / v_{\mathrm{Ai}}$. The key parameters controlling the dispersion properties of the kink waves are the so-called density contrast, $\eta=\rho_{\mathrm{e}} / \rho_{\mathrm{i}}$, defined as the ratio of the density of the environment to that of the twisted tube, the ratio of the two background magnetic fields, $b=B_{\mathrm{e}} / B_{\mathrm{i}}$, the twist of the magnetic field, $\varepsilon$, and, naturally, the mass flow characterised by the Alfvén-Mach number $M_{\mathrm{A}}$.

To study the influence of the mass flow, the twist of the equilibrium magnetic field inside the tube, as well as the environment's magnetic field on the dispersion properties and stability of the kink waves, we considered two cases with fixed density contrasts $\eta=2$ and 0.1 , respectively. For a given $\eta$ and a specified external magnetic field via the parameter $b$, we investigated the kink wave dispersion curves and the instability growth rate when the wave becomes unstable for two different magnetic field 
twist parameters, namely $\varepsilon=0.1$ and 0.4 , respectively, by gradually changing the normalised mass flow velocity, i.e., the AlfvénMach number $M_{\mathrm{A}}$, from zero (rest plasma) to some reasonable values. The flow shifts both the kink-speed curves and the dispersion curves of the wave harmonics upwards. We focused our study on the kink-speed wave only since it is the mode that plays an important role in the dynamics of photospheric and chromospheric/coronal flows.

To evaluate the effect of the adopted magnetic field twist, $\varepsilon$, on the kink-speed wave dispersion curves and chiefly on the value of the critical Alfvén-Mach number that determines that flow speed that ensures the onset of an instability of the KelvinHelmholtz type, we compared the wave dispersion diagrams in twisted tubes with those in untwisted magnetic tubes. It is well established that in untwisted magnetic tubes the kink waves are subject to the Kelvin-Helmholtz instability when $M_{\mathrm{A}}$ exceeds a certain critical value, depending upon the density contrast, $\eta$, and on the ratio of the background magnetic fields, $b$, in both media (see Vasheghani Farahani et al. 2009; Zhelyazkov 2010, 2012a). For an isolated magnetic tube, $b=0$, and, hence, the threshold value of the Alfvén-Mach number depends only on the density contrast, $\eta$. Relatively high values of $\eta$ yield lower threshold Alfvén-Mach numbers while the lower values of $\eta$ do just the opposite - for our chosen two $\eta \mathrm{s}$ the corresponding critical normalised flow speeds are 1.30825 and 3.902503. The effect of the magnetic field twist critically depends on the value of the twist parameter $\varepsilon$. This effect is strongest when $\varepsilon=0.4$ - then one observes a noticeable decrease in the threshold Alfvén-Mach number: for a tube with $\eta=2$ and $b=0, M_{\mathrm{A}}^{\text {cr }}=1.250075$, while at $\eta=0.1$ it is equal to 3.778422 . This substantial finding that the twisted magnetic field lowers the threshold $M_{\mathrm{A}}$ for the occurrence of the Kelvin-Helmholtz instability of $m=1$ mode agrees with the same statement declared in the works by Bennett et al. (1999) and Zaqarashvili et al. (2010). In particular, in twisted photospheric magnetic tubes with a density contrast $\eta=2$, magnetic field twist $\varepsilon=0.4$, and Alfvén speed $v_{\mathrm{A}}=10 \mathrm{~km} \mathrm{~s}^{-1}$, a reliable mass flow of $12.5 \mathrm{~km} \mathrm{~s}^{-1}$ can cause the onset of an instability of the Kelvin-Helmholtz type of $m=1$ mode. Any none-zero environment magnetic field, $B_{\mathrm{e}}$, increases $M_{\mathrm{A}}^{\text {cr }}$, slightly for $b=$ 0.1 , and more noticeable at $b=0.5$. The observation/detection of the Kelvin-Helmholtz instability of $m=1$ mode in a coronal twisted magnetic tube (at $\eta=0.1$ ) is generally problematic, but a twist of 0.4 might help starting this instability in coronal $\mathrm{X}$-ray flows, for which the parameter $\eta$ is slightly bigger (of the order of 0.14), and the observed flow speeds can be much higher, a few thousand kilometers per second (see Madjarska 2011; also Zhelyazkov 2012b, and the discussion therein).

A criterion for the appearance of the Kelvin-Helmholtz instability of kink waves in untwisted magnetic tubes is the satisfaction of an inequality suggested by Andries \& Goossens (2001), which in our notation reads

$M_{\mathrm{A}}>1+\frac{B_{\mathrm{e}} / B_{\mathrm{i}}}{\sqrt{\eta}}$

For twisted magnetic tubes, and in particular for isolated ones (with $B_{\mathrm{e}}=0$ ), this criterion provides a fairly rough prediction, just $M_{\mathrm{A}}>1$. In other words, the mass flows must be superAlfvénic to trigger an instability - this seems to be a norm for twisted magnetic tubes with field-aligned flows (see Díaz et al. 2011). Our numerical findings of the critical Alfvén-Mach numbers also agree with that rough criterion - in both cases $(\eta=2$ and 0.1$)$ the Kelvin-Helmholtz instability occurs when the axial mass flows are super-Alfvénic.
On the other hand, a criterion for the appearance of the Kelvin-Helmholtz instability in isolated twisted magnetic tubes is the satisfaction of an inequality suggested by Zaqarashvili et al. (2010), which in our notation is

$m M_{\mathrm{A}}^{2}>1+1 / \eta$.

For the kink mode, i.e., $m=1$, this inequality gives $M_{\mathrm{A}}>$ 1.2247 for $\eta=2$ and $M_{\mathrm{A}}>3.3166$ for $\eta=0.1$. Our numerical findings of the critical Alfvén-Mach numbers agree with this criterion in both cases.

The next steps in studying the Kelvin-Helmholtz instability of kink waves in twisted magnetic tubes are obviously (i) to investigate the role of the transverse density inhomogeneity inside the tube and associated with it, resonant wave absorption on the kink waves propagation and their stability/instability status; and (ii) to consider a field-aligned mass flow instead of an axial one.

Acknowledgements. I.Zh. thanks the Sofia University Scientific Fund for support under grant No. 147-2012. The work of T.V.Z. was supported by the Austrian Fonds zur Förderung der wissenschaftlichen Forschung (project P21197-N16) and by the European FP7-PEOPLE-2010-IRSES-269299 project-SOLSPANET. The authors thank the anonymous referee for his/her constructive comments and suggestions, which led to significant improvements in the paper.

\section{References}

Acton, F. S. 1990, Numerical Methods That (Usually) Work (Washington, D.C.: Mathematical Association of America), Chap. 14 Andries, J., \& Goossens, M. 2001, A\&A, 368, 1083

Aschwanden, M. J. 2005, Physics of the Solar Corona (Berlin: Springer), Chap. 6 Aschwanden, M. J., Fletcher, L., Schrijver, C. J., \& Alexander, D. 1999, ApJ, 520,880

Bennett, K., Roberts, B., \& Narain, U. 1999, Sol. Phys., 185, 41

Bogdan, T. J. 1984, ApJ, 282, 769

Carter, B. K., \& Erdélyi, R. 2008, A\&A, 481, 239

Chae, J., Wang, H., Qiu, J., et al. 2001, ApJ, 560, 476

Chandrasekhar, S. 1961, Hydrodynamic and Hydromagnetic Stability (Oxford: Clarendon Press), Chap. 11

Cirtain, J. W., Golub, L., Lundquist, L., et al. 2007, Science, 318, 1580 de Pontieu, B., McIntosh, S., Hansteen, V. H., et al. 2007, PASJ, 59, S655

Díaz, A. J., Oliver, R., Ballester, J. L., \& Soler, R. 2011, A\&A, 533, A95

Drazin, P. G. 2002, Introduction to Hydrodynamic Stability (Cambridge: Cambridge University Press), Chap. 8

Dungey, J. W., \& Loughhead, R. E. 1954, Aust. J. Phys., 7, 5

Ebadi, H., Zaqarashvili, T. V., \& Zhelyazkov, I. 2012, Astrophys. Space Sci., 337,33

Edwin, P. M., \& Roberts, B. 1983, Sol. Phys., 88, 179

Erdélyi, R., \& Carter, B. K. 2006, A\&A, 455, 361

Erdélyi, R., \& Fedun, V. 2006, Sol. Phys., 238, 41

Erdélyi, R., \& Fedun, V. 2007, Sol. Phys., 246, 101

Erdélyi, R., \& Fedun, V. 2010, Sol. Phys., 263, 63

Foullon, C., Verwichte, E., Nakariakov, V. M., Nykyri, K., \& Farrugia, C. J. 2011, ApJ, 729, L8

Furno, I., Intrator, T. P., Lapenta, G., at al. 2007, Phys. Plasmas, 14, 022103

He, J., Marsch, E., Tu, C., \& Tian, H. 2009, ApJ, 705, L217

Hood, A. W., \& Priest, E. R. 1979, Sol. Phys., 64, 303

Kamio, S., Peter, H., Curdt, W., \& Solanki, S. K. 2011, A\&A, 532, A96

Karami, K., \& Bahari, K. 2010, Sol. Phys., 263, 87

Karami, K., \& Barin, M. 2009, MNRAS, 394, 521

Katsukawa, Y., Berger, T. E., Ichimoto, K., et al. 2007, Science, 318, 1594

Kruskal, M. D., Johnson, J. L., Gottlieb, M. B., \& Goldman, L. M. 1958, Phys. Fluids, 1, 421

Kukhianidze, V., Zaqarashvili, T. V., \& Khutsishvili, E. 2006, A\&A, 449, L35

Linton, M. G., Longcope, D. W., \& Fisher, G. H. 1996, ApJ, 469, 954

Lundquist, S. 1951, Phys. Rev., 83, 307

Madjarska, M. S. 2011, A\&A, 526, A19

Muller, D. E. 1956, Math. Tables Other Aids Comput., 10, 208

Ofman, L., \& Wang, T. J. 2008, A\&A, 482, L9

Ofman, L., \& Thompson, B. J. 2011, ApJ, 734, L11

Pietarila, A., Aznar Cuadrado, R., Hirzberger, J., \& Solanki, S. K. 2011, ApJ, 739, 92

Roberts, P. H. 1956, ApJ, 124, 430 
I. Zhelyazkov and T. V. Zaqarashvili: Kelvin-Helmholtz instability of kink waves in twisted magnetic tubes

Ruderman, M. S. 2007, Sol. Phys, 246, 119

Ruderman, M. S., \& Erdélyi, R. 2009, Space Sci. Rev., 149, 199

Shafranov, V. D. 1957, J. Nuclear Energy II, 5, 86

Shibata, K., Nakamura, T., Matsumoto, T., et al. 2007, Science, 318, 1591

Shimojo, M., \& Shibata, K. 2000, ApJ, 542, 1100

Soler, R., Terradas, J., \& Goossens, M. 2010a, ApJ, 734, 80

Soler, R., Terradas, J., Verth, G., \& Goossens, M. 2010b, ApJ, 736, 10

Srivastava, A. K., \& Murawski, K. 2011, A\&A, 534, A62

Srivastava, A. K., Zaqarashvili, T. V., Kumar, P., \& Khodachenko, M. L. 2010, ApJ, 715, 292

Tavabi, E., Koutchmy, S., \& Ajabshirizadeh, A. 2011, New Astron., 16, 296

Terradas, J., Andries, J., Goossens, M., et al. 2008, ApJ, 687, L115

Terradas, J., Goossens, M., \& Ballai, I. 2010a, A\&A, 515, A46

Terradas, J., Goossens, M., \& Verth, G. 2010b, A\&A, 524, A23

Tian, H., McIntosh, S. W., Rifal Habbal, S., \& He, J. 2011, ApJ, 736, 130

Trehan, S. K. 1958, ApJ, 127, 446

Ugarte-Urra, I., Doyle, J. G., Walsh, R. W., \& Madjarska, M. S. 2005, A\&A, 439,351
Vasheghani Farahani, S., Van Doorsselaere, T., Verwichte, E., \& Nakariakov, V. M. 2009, A\&A, 498, L29

Verwichte, E., Aschwanden, M. J., Van Doorsselaere, T., Foullon, C., \& Nakariakov, V. M. 2009, ApJ, 698, 397

Wallace, A. J., Harra, L. K., van Driel-Gesztelyi, L., Green, L. M., \& Matthews, S. A. 2010, Sol. Phys., 267, 361

Zaqarashvili, T. V. 2011, in 3rd School and Workshop on Space Plasma Physics, eds. I. Zhelyazkov, \& T. Mishonov, AIP Conf. Proc., 1356, 106

Zaqarashvili, T. V., \& Erdélyi, R. 2009, Space Sci. Rev., 149, 355

Zaqarashvili, T. V., Khutsishvili, E., Kukhianidze, V., \& Ramishvili, G. 2007, A\&A, 474, 627

Zaqarashvili, T. V., Díaz, A. J., Oliver, R., \& Ballester, J. L. 2010, A\&A, 516, A84

Zhelyazkov, I. 2010, Plasma Phys. Control. Fusion, 52, 065008

Zhelyazkov, I. 2012a, A\&A, 537, A124

Zhelyazkov, I. 2012b, in Topics in Magnetohydrodynamics, ed. L. Zheng, InTech - Open Access Company, Chap. 6 